\title{
PROCEDIMENTO PARA ESCOLHA DE POPULAÇÕES DE MILHO PROMISSORAS PARA EXTRAÇÃO DE LINHAGENS $^{(1)}$
}

\author{
MAX WENDEL PAULA LIMA ${ }^{(2)}$; ELAINE APARECIDA DE SOUZA ${ }^{(2)}$; \\ MAGNO ANTONIO PATTO RAMALHO ${ }^{(2)}$
}

\begin{abstract}
RESUMO
O sucesso de um programa de melhoramento de milho visando à obtenção de híbridos está intimamente ligado à identificação da população mais promissora para a extração de linhagens. $\mathrm{O}$ presente trabalho objetivou identificar procedimentos para a escolha dessas populações. Para isso, foram obtidas populações segregantes $S_{0}, S_{1}$ e 196 famílias $S_{0: 1}$ de cada um dos quatro materiais comerciais avaliados: híbridos simples (C 333B e Z 8392), duplo (AG 1051) e variedade (BR-105). Os experimentos foram conduzidos na safra agrícola $98 / 99$ em duas localidades na região sul do Estado de Minas Gerais: Lavras e Ijaci. Na avaliação das 196 famílias $S_{0: 1}$ de cada população foi empregado o delineamento látice simples $14 \times 14$. Adicionalmente foi instalado um experimento em blocos casualizados, com 4 repetições, para avaliação simultânea das gerações $F_{1}, S_{0}$ e $S_{1} A$ partir dos dados de produtividade de espigas despalhadas (kg por parcela) das gerações $F_{1}, S_{0}$ e $S_{1}$, foram obtidas as estimativas da contribuição dos locos em homozigose $(\mathbf{m}+\mathbf{a})$ e em heterozigose $(\mathbf{d})$. Foram também foram estimados os parâmetros genéticos e fenotípicos com os experimentos das famílias $\mathrm{S}_{0: 1}$. Constatou-se que houve boa associação $(r=0,81)$ entre a estimativa de $(\mathbf{m}+\mathbf{a})$ e a média das famílias $\mathrm{S}_{0: 1} \mathrm{e}$ que população com maior potencial para a extração de linhagens, maior $(\mathbf{m}+\mathbf{a})$, foi a AG 1051. A correlação entre as estimativas de (d) $\mathrm{e} \mathrm{h}^{2}$ foi baixa, indicando que a estimativa da contribuição dos locos em heterozigose não foi bom indicador da variabilidade potencial da população.

Palavras-chave: milho, linhagem, depressão por endogamia, $\mathbf{m}+\mathbf{a}$, variabilidade, populações.
\end{abstract}

\section{ABSTRACT \\ PROCEDURE TO SELECT SUPERIOR MAIZE POPULATIONS FOR INBRED LINE EXTRACTION}

The identification of the population with greatest potential for inbred line extraction is directly linked to the success of a maize hybrid breeding program. This study was carried out to identify procedures for selecting these populations. Segregant $\mathrm{S}_{0}, \mathrm{~S}_{1}$ populations and $196 \mathrm{~S}_{0: 1}$ families were obtained from each of four commercial cultivars assessed: single hybrids (C 333B and Z8392), double hybrid (AG 1051) and variety (BR-105). The experiments were carried out during the 1998/99 season, in two locations in the southern region of Minas Gerais State, Brazil: Lavras and Ijaci. A 14 x 14 simple lattice design was used in the assessment of the $196 \mathrm{~S}_{0: 1}$ families from each population. A randomized complete blocks design with four replications experiment was also set up to assess, simultaneously, the $F_{1}, S_{0}$ and $S_{1}$ generations. The husked ear yield (kg/plot) of the $F_{1}, S_{0}$ and $S_{1}$ generations was obtained and the contribution of the homozygous locos $(\mathbf{m}+\mathbf{a})$ and heterozygous locos $(\mathbf{d})$ were

$\left.{ }^{1}\right)$ Parte da dissertação de mestrado do primeiro autor apresentada à Universidade Federal de Lavras (UFLA) em 5 de agosto de 1999. Recebido para publicação em 18 de novembro de 1999 e aceito em 9 de maio de 2000.

${ }^{2}$ ) Departamento de Biologia da Universidade Federal de Lavras, Caixa Postal 37, 37200-000 Lavras (MG). 
estimated. The genetic and phenotypic parameters were also estimated using the $\mathrm{S}_{0: 1}$ family experiments. A high correlation $(r=0.81)$ between the $(\mathbf{m}+\mathbf{a})$ estimate and the $S_{0: 1}$ family mean was observed. The AG 1051 population showed the greatest potential for line extraction, i.e., greatest $(\mathbf{m}+\mathbf{a})$ value. The correlation between the $\mathrm{d}$ and $\mathrm{h}^{2}$ estimates was low, indicating that the estimate of the contribution of the heterozygous locos was not a good indicator of the potential variability of the population. Key words: maize, line, endogamy depression, $(\mathrm{m}+\mathrm{a})$, variability, populations.

\section{INTRODUÇÃO}

O sucesso de um programa de melhoramento de milho visando à obtenção de híbridos está ligado à identificação da população mais promissora para a extração de linhagens. Entre os procedimentos que auxiliam os melhoristas nessa escolha, destaca-se a estimativa de $\mathbf{m}+\mathbf{a}$, que corresponde à média de todas as linhagens na geração $S_{\infty}$. Esse método proposto por VENCOVSKY (1987) permite avaliar o potencial das populações em gerações precoces, estimando-se $\mathbf{m}+\mathbf{a}$ por meio do contraste entre as gerações $S_{0}$ e $S_{1}$. O procedimento tem sido utilizado no Brasil, em algumas oportunidades (VIANNA et al.,1982; LIMA et al., 1984; TERASAWA JunIOR, 1993; Nass e Miranda Filho, 1995; PaCKer, 1998; SOUZa SObrinho et al., 1998), quando se constatou variação entre as populações.

Vale ressaltar, contudo, que apenas a média das linhagens na geração $S_{\infty}$ não é suficiente para se avaliar o potencial das populações. Para que a seleção possa ser efetuada, há necessidade da existência de variação entre as linhagens. Assim, é importante utilizar procedimentos que possibilitem quantificar essa variação nas populações o mais precocemente possível. Uma das alternativas seria a estimativa da variância genética entre plantas, na população original. Esse procedimento é pouco prático, devido aos erros associados às estimativas de variância para cada planta, individualmente, e à dificuldade de se obterem informações por planta.

Quanto maior a freqüência de locos em heterozigose, na população, maior a possibilidade de se obterem linhagens de constituição genética diferente na geração $S_{\infty}$. Havendo dominância no controle do caráter alvo, bem como locos em heterozigose, pode-se prever que ocorra depressão por endogamia (DE) na população. A partir do exposto, infere-se que a estimativa da depressão por endogamia pode indicar a contribuição dos locos em heterozigose (d), na população. Assim, na comparação entre duas populações, desde que a contribuição dos diferentes locos seja semelhante, terá maior depressão por endogamia a população com maior freqüência de locos em heterozigose e, por conseguinte, maior liberação de variabilidade será esperada entre as linhagens. Tal procedimento já foi utilizado em plantas autógamas (ABREU, 1997), porém, no caso específico do milho são restritas as informações a esse respeito.

Nos programas de melhoramento visando à obtenção de linhagens, uma das alternativas é a utilização de híbridos comerciais como fonte de novas linhagens - denominadas linhagens de segundo ciclo - sendo essa uma prática comum entre os melhoristas (Hallauer, 1990; Lamkey et al., 1995). Como há disponibilidade de algumas dezenas de híbridos comerciais seria importante identificar estratégias que possam ser utilizadas na escolha dos híbridos e/ou populações segregantes como fontes de novas linhagens.

Assim, este trabalho objetivou detectar a contribuição dos locos em heterozigose e da estimativa de $\mathbf{m}+\mathbf{a}$ como nova alternativa na escolha das populações a serem utilizadas como fontes de novas linhagens.

\section{MATERIAL E MÉTODOS}

O ensaios foram conduzidos na área experimental do Departamento de Biologia da Universidade Federal de Lavras (UFLA) e na fazenda da FAEPE, município de Ijaci (MG), durante a safra 98/99. Foram utilizadas quatro cultivares, sendo dois híbridos simples (C 333B e Z 8392), um híbrido duplo (AG 1051) e uma variedade (BR-105).

As gerações $S_{0}=F_{2}$ dos híbridos simples e duplo foram obtidas a partir de polinização livre das plantas $\mathrm{F}_{1}$, em campos isolados. Parte das sementes obtidas foi armazenada e o restante, juntamente com as sementes $S_{0}$ da variedade BR 105, foi semeado para obtenção da geração $S_{1}$ e das famílias $S_{0: 1}$. $O$ número mínimo de plantas para cada material foi 700, e, nesse total, ocorreram pelo menos 500 autofecundações.

Por ocasião da colheita foram tomadas as espigas de cada planta autofecundada, individualmente. Das espigas obtidas foram tomadas, aleatoriamente, 196 famílias $\mathrm{S}_{0: 1}$ por material, sendo retiradas de cada uma delas 10 sementes que, posteriormente, foram misturadas para constituir a população $S_{1}$ de cada material. 
As avaliações foram realizadas nos dois locais mencionados. Para cada população foi conduzido um experimento distinto para avaliação das 196 famílias $\mathrm{S}_{0: 1}$, empregando-se o delineamento de látice simples $14 \times 14$. As parcelas foram constituídas por uma linha de $3 \mathrm{~m}$ espaçada de $0,90 \mathrm{~m}$, onde foram semeadas 10 sementes por metro linear.

Em Lavras foram também avaliadas, simultaneamente, as gerações $S_{0}$ e $S_{1}$ das quatro populações e a geração $F_{1}$ dos híbridos, em um experimento, utilizando-se o delineamento em blocos casualizados, com 11 tratamentos e quatro repetições. Nesse caso, as parcelas foram compostas por quatro linhas de $5 \mathrm{~m}$, espaçadas de $0,90 \mathrm{~m}$, colocando-se 10 sementes por metro linear. Procedeu-se ao desbaste em todos os experimentos, permanecendo cinco plantas por metro linear. Os tratos culturais foram os normais para a cultura da região.

Foram anotados os dados de peso de espigas despalhadas (kg por parcela), a partir dos quais realizaram-se análises individuais e conjunta de variância. Foram obtidas estimativas dos componentes de médias $\mathbf{m}+\mathbf{a}$ e $\mathbf{d}$, utilizando-se procedimento semelhante ao apresentado por VeNCOVSKY (1987). Em vista de existirem, nesse caso, mais de dois tipos de populações, as estimativas de $\mathbf{m}+\mathbf{a}$ e $\mathbf{d}$ foram obtidas pelo método dos quadrados mínimos, tendo como referência a população $\mathrm{S}_{0}$. Desse modo, $\overline{\mathrm{S}}_{\mathrm{i}_{\mathrm{i}}}=\mathrm{m}+\mathrm{a}_{\mathrm{i}}+\mathrm{d}_{\mathrm{i}}$, $\overline{\mathrm{S}}_{1_{\mathrm{i}}}=\mathrm{m}+\mathrm{a}_{\mathrm{i}}+1 / 2 \mathrm{~d}_{\mathrm{i}}$ e $\overline{\mathrm{F}}_{1_{\mathrm{i}}}=\mathrm{m}+\mathrm{a}_{\mathrm{i}}+2 \mathrm{~d}_{\mathrm{i}}$, em que $: \overline{\mathrm{F}}_{1_{\mathrm{i}}}, \overline{\mathrm{S}}_{0_{\mathbf{i}}}$ e $\bar{S}_{1_{i}}$ são as médias das gerações $F_{1}, S_{0}$ e $S_{1}$, respectivamente, da população i; $\mathbf{m}+\mathbf{a}$ é a contribuição dos locos em homozigose; e $\mathbf{d}$ é a contribuição dos locos em heterozigose, para a média do caráter.

A partir das análises de variância individuais e conjunta para todas as populações foram obtidas as estimativas de herdabilidade no sentido amplo $\left(\mathrm{h}^{2}\right)$, utilizando-se o método apresentado por VENCOVSKY e BARRIGA (1992) e seus respectivos intervalos de confiança (LI: limite inferior e LS: limite superior para $1-\boldsymbol{\alpha}=0,95)$, de acordo com o método de KNAPP et al. (1985).

\section{RESULTADOS E DISCUSSÃO}

Verificou-se diferença na produtividade média das diversas gerações dentro de cada população avaliada (Quadro 1). As maiores produtividades foram obtidas para a geração $F_{1}$ dos híbridos $C$ 333B e AG 1051. Na geração $S_{0}$, o melhor desempenho foi obtido pelo híbrido AG 1051, destacando-se também a variedade BR105. Vale ressaltar que a média da geração $S_{1}$ foi muito semelhante à média geral das famílias $S_{0: 1}$, para todas as populações.
Quadro 1. Produtividade média de espigas despalhadas em t.ha ${ }^{-1}$, das gerações $F_{1}, S_{0}$ e $S_{1}$, famílias $S_{0: 1}$ e estimativas dos componentes de média $(\mathrm{m}+\mathrm{a}$ e $\mathrm{d})$ e seus respectivos erros em quatro populações de milho, em Lavras (MG). Safra 98/99

\begin{tabular}{lcccc} 
& \multicolumn{4}{c}{ Populações } \\
\cline { 2 - 5 } Gerações & C 333B & Z 8392 & AG 1051 & BR-105 \\
\cline { 2 - 5 } & \multicolumn{4}{c}{ Produtividade (t.ha $\left.{ }^{-1}\right)$} \\
\hline $\mathrm{F}_{1}$ & 13,415 & 11,093 & 13,140 & - \\
$\mathrm{S}_{0}$ & 8,731 & 6,931 & 11,667 & 10,082 \\
$\mathrm{~S}_{1}$ & 6,691 & 5,719 & 7,252 & 6,987 \\
$\mathrm{~S}_{0: 1}$ & 6,780 & 6,091 & 6,956 & 6,489 \\
\hline \multicolumn{4}{c}{ Componentes de média (t.ha $\left.{ }^{-1}\right)$} \\
\hline m + a & $4,34(0,54)$ & $3,64(0,54)$ & $6,51(0,54)$ & $3,89(0,98)$ \\
d & $4,51(0,41)$ & $3,66(0,41)$ & $3,57(0,41)$ & $6,19(1,24)$ \\
\hline
\end{tabular}

As estimativas de $\mathbf{m}+\mathbf{a}$ variaram de 3,64 t.ha $^{-1}$ para o híbrido Z 8392 a 6,51 t.ha ${ }^{-1}$ para AG 1051, cuja estimativa diferiu significativamente das demais pelo teste $t$ (Quadro 1). A partir do exposto, pode-se inferir que a média das linhagens na geração $S_{\infty}$ do híbrido AG 1051 será superior às demais. O resultado evidencia que esse híbrido, uma vez utilizado em programa de extração de linhagens, irá produzir linhagens que serão, em média, $79 \%$ e $67 \%$ superiores à média prevista para as demais linhagens do híbrido simples $Z$ 8392 e da variedade BR105. Fica evidenciado também que a elevada produtividade desse híbrido, na geração $F_{1}$, deve, pelo menos em parte, ser atribuída à boa produtividade das linhagens genitoras. A informação é bastante útil, visto que possibilita avaliar o potencial da população, para a extração de linhagens, e tem sido freqüentemente utilizada na cultura do milho (VIANA et al. 1982; NAss e Miranda Filho, 1995; PACKer, 1998) e de outras espécies, como o feijão (Oliveira, 1995; ABreu, 1997).

A correlação entre a estimativa de $\mathbf{m}+\mathbf{a}$ e a média das famílias $\mathrm{S}_{0: 1}$ de cada população foi alta $(\mathrm{r}=0,81)$, indicando que $\mathbf{m}+\mathbf{a}$ representa adequadamente a média das famílias $\mathrm{S}_{0: 1}$. Tal resultado reforça as observações anteriores sobre a importância da estimativa de $\mathbf{m}+\mathbf{a}$, embora a média das famílias $S_{0: 1}$ não represente plenamente a das linhagens na geração $S_{\infty}$, visto que existem ainda inúmeros locos em heterozigose. $\mathrm{O}$ referido resultado constitui um indicativo do potencial da população para a extração de linhagens. No caso do milho não foi encontrado nenhum resultado a esse respeito. Contudo, para o feijoeiro, a correlação de $\mathbf{m}+\mathbf{a}$ e a média de famílias $\mathrm{F}_{5: 7}$ (estando basi- 
camente todos os locos em homozigose) correpondeu a 0,97 (ABREu, 1997), realçando o que foi comentado anteriormente e validando o modelo genético adotado, sem epístase.

A estimativa de $\mathbf{d}$ (contribuição dos locos em heterozigose) ao contrário do previsto, foi maior na população BR105 ( $\mathrm{d}=6,19$ t.ha $\left.{ }^{-1}\right)$. Quanto aos híbridos, as estimativas foram semelhantes (Quadro 1). Vale ressaltar que, por meio da estimativa de d, é possível comprovar a contribuição da heterose para o desempenho dos híbridos. Assim, no caso de C333B, a contribuição da heterose atingiu $67,24 \%$, ou seja (2 $x$ 4,51)/13,41, dado que a estimativa de $\mathbf{d}$ tem como referência a população $S_{0}$, e, portanto, a heterose da geração $\mathrm{F}_{1}$ contém 2 d. Em AG 1051 a contribuição da heterose foi menor: $54,30 \%$.

A estimativa de $\mathbf{m}+\mathbf{a}$, embora bastante útil, não possibilita deduzir a variabilidade esperada entre as linhagens. Dessa forma, duas populações podem dar origem a linhagens com a mesma média; no entanto, a amplitude de variação no desempenho dessas linhagens pode ser bastante diferente. É evidente a preferência pela população que permita obter linhagens que apresentem entre si maior amplitude de variação. A variância fenotípica presente em gerações precoces é um indicativo da variabilidade potencial entre as linhagens, na geração $S_{\infty}$. A estimativa da variância fenotípica deve ser obtida a partir de dados de plantas individuais. Contudo, esse fato traz, entre outros problemas, a baixa precisão da estimativa e a dificuldade na obtenção dos dados. Uma alternativa seria obter a estimativa da contribuição dos locos em heterozigose (d), visto que, a partir do pressuposto de que todos os locos têm a mesma contribuição para o fenótipo, a maior estimativa de $\mathbf{d}$ implica maior freqüência de locos em heterozigose. Desse modo, maior variância será esperada entre as linhagens, uma vez que o número de linhagens diferentes em $S_{\infty}$ é dado por $2^{\mathrm{n}}$, em que n é o número de locos segregantes. Sendo correta essa pressuposição, a estimativa de $\mathbf{d}$ irá refletir a variância potencial a ser liberada na geração $S_{\infty}$ e, tratando-se de um componente de média, é válido esperar maior precisão em sua estimativa. Ademais, é menor o trabalho envolvido na sua obtenção.

Para comprovar tal fato, seria necessário comparar a estimativa de $\mathbf{d}$ com a variância genética entre as linhagens, na geração $S_{\infty}$; entretanto, como não foi possível obter essa variância, utilizou-se a variação liberada entre as famílias $\mathrm{S}_{0: 1}$ como indicador do potencial de variabilidade genética das populações, em gerações mais avançadas. A alternativa tem como principal vantagem a redução do erro amostral que, certamente, ocorreria em gerações mais avançadas, pois haveria necessidade de se avaliar um número crescente de famílias, a cada geração de autofecundação.

Para melhor comparação entre as populações optou-se por utilizar a estimativa de herdabilidade $\left(\mathrm{h}^{2}\right)$ em lugar da variância genética entre as famílias. É necessário, inicialmente, enfatizar que, no caso do híbrido duplo e da variedade, a $\mathrm{h}^{2}$ entre famílias $\mathrm{S}_{0: 1}$ contém, no numerador da sua expressão $\sigma^{2}{ }_{A}+1 / 4 \sigma^{2} D$ $+\mathrm{D}_{1}+1 / 8 \mathrm{D}_{2}$ (CoCKerham e Matzinger, 1985; Souza JúNIOR, 1989), pois a freqüência alélica não deve ser 0,5 , apresentando além de $\sigma_{\mathrm{A}}^{2}$ e $\sigma^{2} \mathrm{D}$ próprias do híbrido simples, também os componentes $\mathrm{D}_{1}$ (covariância entre os efeitos aditivos e dominantes dos homozigotos) e $\mathrm{D}_{2}$ (variância dos efeitos de dominância nos homozigotos). Assim, a herdabilidade entre famílias $S_{0: 1}$ superestima a herdabilidade esperada na geração $\mathrm{S}_{\infty}$. Isso se visualiza, facilmente, considerando-se que na geração $S_{0: 1}$ existe ainda grande número de locos em heterozigose que irão segregar posteriormente, ampliando a variabilidade. Entretanto, há expectativa de que populações com maior herdabilidade na geração $S_{0: 1}$ também o sejam, em $S_{\infty}$.

As estimativas de $h^{2}$ são apresentadas no quadro 2. Embora existam algumas discrepâncias nas estimativas entre os locais, em todos eles as estimativas dos limites inferior e superior possibilitam inferir que foram diferentes de zero, ou seja, há variação genética entre as famílias. Utilizando a $\mathrm{h}^{2}$ obtida na análise conjunta é possível concluir que os híbridos C 333B e AG 1051 foram os que apresentaram maior estimativa de herdabilidade. Ressalta-se, contudo, que os valores das outras duas populações foram também moderados. Em princípio, isso reforça a observação anterior de que nos híbridos e na variedade o número de locos segregantes é elevado e, portanto, a variabilidade genética liberada é expressiva. As estimativas de $\mathrm{h}^{2}$ obtidas foram de magnitude semelhante às relatadas na literatura (GROMBACHER et al. 1989; PINTO, 1996; PACKER, 1998).

A estimativa da correlação entre $\mathbf{d} \mathrm{e}^{2}$ envolvendo os experimentos de Lavras foi baixa $(r=0,23)$, indicando que a estimativa de $\mathbf{d}$ não forneceu a mesma informação da herdabilidade, não sendo, portanto, uma boa medida da variabilidade existente na população. Esse resultado foi discrepante do alcançado por ABReu (1997) que, trabalhando com quatro populações de feijão, obteve uma correlação alta e positiva $(\mathrm{r}=0,97)$ entre as estimativas de $\mathbf{d}$ e $\mathrm{h}^{2}$ das famílias $\mathrm{F}_{5: 7}$. Uma explicação para a baixa correlação foi devido ao fato de que podem estar envolvidos locos 
Quadro 2. Estimativas das herdabilidades, no sentido amplo $\left(\mathrm{h}^{2} \%\right)$, em nível de médias de famílias por local e conjunta e seus respectivos intervalos de confiança, limites inferior (LI) e superior (LS) da produção de espigas despalhadas (kg por parcela)

\begin{tabular}{|c|c|c|c|c|}
\hline \multicolumn{5}{|c|}{ Populações } \\
\hline & C 333B & Z 8392 & AG 1051 & BR-105 \\
\hline & \multicolumn{4}{|c|}{ Lavras } \\
\hline$h^{2}$ & 70,22 & 40,21 & 55,43 & 53,91 \\
\hline LI & 60,17 & 20,04 & 40,94 & 38,37 \\
\hline \multirow[t]{2}{*}{ LS } & 78,80 & 55,43 & 66,37 & 65,54 \\
\hline & \multicolumn{4}{|c|}{ Ijaci } \\
\hline$h^{2}$ & 52,82 & 33,51 & 55,92 & 37,75 \\
\hline LI & 36,91 & 11,08 & 41,05 & 16,75 \\
\hline \multirow[t]{2}{*}{ LS } & 64,83 & 50,44 & 67,14 & 53,59 \\
\hline & \multicolumn{4}{|c|}{ Conjunta } \\
\hline$h^{2}$ & 69,20 & 43,90 & 59,10 & 45,70 \\
\hline LI & 59,19 & 25,66 & 45,79 & 27,98 \\
\hline LS & 76,76 & 57,67 & 69,13 & 58,99 \\
\hline
\end{tabular}

com diferentes contribuições, isto é, em alguns cruzamentos, embora a estimativa de $\mathbf{d}$ tenha sido grande, um número restrito de locos foi responsável pelo vigor observado. Nesse caso, o modelo adotado não seria adequado, visto considerar que os locos têm o mesmo efeito.

\section{CONCLUSÕES}

1. A população $S_{0}$ com maior potencial para a extração de linhagens é a do híbrido duplo AG 1051, tendo apresentado alta estimativa de $\mathbf{m}+\mathbf{a}$.

2. A correlação entre as estimativas de $\mathbf{d ~ e ~} \mathrm{h}^{2}$ foi baixa, indicando que a estimativa da contribuição dos locos em heterozigose não se caracterizou como bom indicador da variabilidade potencial da população.

\section{REFERÊNCIAS BIBLIOGRÁFICAS}

ABREU, A. de F.B. Predição do potencial genético de populações segregantes do feijoeiro utilizando genitores inter-raciais. Lavras, 1997. 80p. Tese (Doutorado em Genética e Melhoramento de Plantas) - UFLA.

COCKERHAM, C.C.; MATZINGER, D.F. Selection response based on selfed progenies. Crop Science, Madison, v.25, p.483-488, 1985.

GROMBACHER, A.W.; RUSSEL, W.A.; GUTHRIE, W.D. Effects of recurrent selection in two maize synthetics on agronomic trait of $\mathrm{S}_{1}$ lines. Maydica, Bergamo, v.4, n.4, p.343-352, 1989.

HALLAUER, A. R. Methods used in developing maize inbreed lines. Maydica, Bergamo, v.35, p.1-16, 1990.
KNAPP, S.J.; STROUP, N.W.; ROSS, W M. Exact confidence intervals for heritability on a progeny mean basis. Crop Science, Madison, v.25, p.192-194, 1985.

LAMKEY, K.R.; SCHNICKER, B.J.; MELCHINGER, A E. Epistasis in an elite maize hybrid and choice of generation for inbred line development. Crop Science, Madison, v.35, n.5, p.1272-1281, 1995.

LIMA, M.; MIRANDA FILHO, J.B.; GALLO, P.B. Inbreeding depression in brazilian populations of maize (Zea mays L.). Maydica, Bergamo, v.29, p.203-215, 1984.

NASS, L. L.; MIRANDA FILHO, J. B. Inbreeding depression rates of semi-exotic maize (Zea mays L.) populations. Revista Brasileira de Genética, Ribeirão Preto, v.18, p.585-592, 1995.

OLIVEIRA, L. B. Alternativas na escolha dos parentais em um programa de melhoramento de feijoeiro. Lavras, 1995.67p. Dissertação (Mestrado em Genética e Melhoramento de Plantas) - UFLA.

PACKER, D. Variabilidade genética e endogamia em quatro populações de milho (Zea mays L.). Piracicaba, 1998. 102p. Tese (Doutorado em Genética e Melhoramento de Plantas) - ESALQ-USP.

PINTO, R.MC. Tamanho da amostra para seleção recorrente com progênies $S_{1}$ em milho. Piracicaba, 1996.92p. Dissertação (Mestrado em Genética e Melhoramento de Plantas) ESALQ-USP.

SOUZA JÚNIOR, C. L. Componentes da variância genética e suas implicações no melhoramento vegetal. 1.ed. Piracicaba, FEALQ, 1989. 134p.

SOUZA SOBRINHO, F. S.; RAMALHO, M. A. P.; SOUZA, J. C. Heterose de alguns híbridos em uso na Região 
Sudeste. In: CONGRESSO NACIONAL DE MILHO E SORGO, 22., Recife, 1998. Resumos expandidos... (CDRom) Recife, ABMS/IPA/EMBRAPA MS, 1998.

TERASAWA JUNIOR, F. Seleção recorrente com endogamia em duas populações de milho: avaliação quantitativa e perspectivas para seleção de híbridos. Piracicaba, 1993. 169p. Dissertação (Mestrado em Genética e Melhoramento de Plantas) - ESALQ-USP.

VENCOVSKY, R. Herança quantitativa. In: PATERNIANI, E.; VIÉGAS, G.P. (Eds.). Melhoramento e produção do milho no Brasil. 2.ed. Campinas: Fundação Cargill, 1987. p.122-201.

VENCOVSKY, R.; BARRIGA, P. Genética biométrica no fitomelhoramento. 1.ed. Ribeirão Preto, Sociedade Brasileira de Genética, 1992. 486p.

VIANNA, R. T.; GAMA, E. E. G.; NASPOLINI FILHO, V.; MORO, J. R.; VENCOVSKY, R. Inbreeding depression of several introduced populations of maize (Zea mays L.). Maydica, Bergamo, v.27, n.1, p.151-157, 1982. 